\title{
MISCELÁNEA
}

\section{UNA CARTA DEL MARQUÉS DE SANTILLANA}

No decimos nada nuevo si comenzamos las siguientes líneas señalando que el Marqués de Santillana es una de las personalidades más atractivas de nuestra Edad Media. Figura de primer orden en la historia politica y cultural del siglo xv español, Iñigo López de Mendoza despertó una gran admiración no sólo en su época sino también entre las generaciones posteriores. Algunos testimonios, como el de Juan de Mena en su Coronación, ponen de manifiesto hasta qué punto era conocido dentro y fuera de España:

Quos ad contemplationem eius Roma non traxit unius hominis fama perduxit de ultimis Hispaniae Galliaque finibus,

añadiendo inmediatamente:

Creý esta palabra poderse decir... del prudentíssimo, magnánimo e ingente cavallero ıñigo López de Mendoça: a la fama del qual muchos extrangeros, que en España non avían causa de passar, ayan por huéspedes sufrido venir en la castellana región, non es a nosotros nuevo. La qual volante fama con alas de ligereza, que son gloria de buenas nuevas, ha encavalgado los gállicos Alpes e discurrió hasta la frigiana tierra 1.

Así pues, el Marqués tenía una personalidad suficientemente atractiva como para hacer venir a España a gentes de otros puntos de Europa. Aunque el testimonio anterior sea un tanto exagerado por el carácter

1 Cito el texto de la Coronación por J. AMADOR DE los Rios, Obras de don Iñigo López de Mendoza, Marqués de Santillana, Madrid, 1852, pág. 128. 
de la obra, hay que considerar sin embargo que el papel de guía que Santillana desempeñó a lo largo de su vida informa suficientemente acerca de su influjo sobre ciertos grupos e individuos. Jóvenes y menos jóvenes piden consejo al Marqués cuando se interesan por la literatura, y éste manda sus obras a cuantos familiares y amigos se las solicitan. Así, su sobrino Gómez Manrique le rogaba el envío de un cancionero por medio de una poesía:

Lo qual mi cobdiçia non faze menor de aver vuestras obras en vn Cançionero?

Esta labor educadora y de difusión se veía complementada por otra parte por su propio interés por la literatura y los libros; así, Santillana llegó a formar un verdadero círculo cultural. El encargo de copias manuscritas a países como Italia y la solicitud de traducciones de textos en latín a personas allegadas serán dos de las tareas desarrolladas de mayor importancia. De esta manera, al escribir a su hijo Pero González de Mendoza su conocida carta "quando estava estudiando en Salamanca», se enorgullece diciendo:

A ruego e instançia mía, primero que de otro alguno, se han vulgariçado en este reyno algunos poemas, asý como la Eneyda de Virgilio, el libro mayor de las Transformaçiones de Ovidio, las Tragedias de Lucio Anio Séneca, e muchas otras cosas, en que yo me he deleytado fasta este tiempo e me deleyto... ${ }^{3}$.

A su propio hijo le rogaba también en esta carta una traducción de la Iliada del latín al castellano ${ }^{4}$. Otras obras de temas diferentes fueron traducidas igualmente por encargo del Marqués, siendo muy posible que la lista actualmente conocida pueda incrementarse con algún nuevo título ${ }^{5}$.

2 Editada por AMADOR DE LOS Ŕ́os, también se encuentra en el Cancionero Castellano del siglo XV de Foulche Delbosc, Madrid, Bailly-Bailliere, 1915, tomo II, pág. 26.

3 AMADOR DE LOS Rfos, op. cit., pág. 482.

4 Demasiadas líneas se han dedicado al conocimiento que el Marqués tenía del latín. Las opiniones oscilan entre un buen conocimiento de la lengua latina -como ocurre por ejemplo con M. GaRCI-Gómez, «Paráfrasis de Cicerón en la definición de poesía de Santillanan, Hispania, LVI (1973), págs. 207-212- y el desconocimiento de dicha lengua -buen ejemplo de ello ofrece Mario SchIrp en su conocida obra La bibliothèque du Marquis de Santillane, Paris, Bouillon, 1905, págs. LXIII-LXIX-, aunque la postura más lógica y comúnmente aceptada es la de un conocimiento relativo de dicha lengua. La prueba de que el encargo del Marqués a su hijo se cumplió parece constituirla un manuscrito de la British Library que contiene la traducción de la obra y un prólogo en castellano que muy probablemente está dirigido al Marqués.

5 Vid. v. g. las páginas que dedica al tema R. LAPESA, La obra literaria del Mar- 
Nuestra atención, sin embargo, se centra ahora sobre sus propias obras y el interés que despertaron en sus días. Otros personajes, al igual que Gómez Manrique, le pidieron envío de sus poesías; de estas peticiones conocemos la de doña Violante de Prades, Condesa de Modica, a quien el Marqués dirigió una carta junto con sus poemas. En este interesante texto, como hará en otros, Santillana comenta y hace referencia a diversos elementos de teoría poética, aspecto en el que quizá reside su mayor importancia. En dicha carta el Marqués hace comentarios sobre algunas obras que ha mandado a la Condesa anteriormente, otras que le manda ahora, y deja abierta la posibilidad de nuevos envíos con palabras no demasiado concretas por su formalismo:

E sy algunas otras cosas, muy noble Señora, vos plazen que yo por honor vuestro e de la casa vuestra faga, con ynfallible fiuza vos pido por merçed asý commo a menor hermano, me escriuades 6 .

El Prohemio que el Marqués mandó al joven Condestable don Pedro de Portugal es, sin duda alguna, la más importante de estas cartas-pró$\operatorname{logos}{ }^{7}$; en dicha obra se responde igualmente a dos necesidades - como ocurría en el texto anterior-, acompañar el envío de un grupo de poemas y ser una especie de guía literaria y estética del joven Condestable ${ }^{8}$.

En la misma línea se encuentra el texto que ahora presentamos. Se trata de una carta que el Marqués envió a su sobrino Pero de Mendoza, Señor de Almazán, en la que, a la vez que le introduce los textos que le envía, hace unas pequeñas pero interesantes referencias a ciertos aspectos literarios y estéticos importantes en ese momento. Unimos a esta carta la de su sobrino por encontrarse juntas en el cancionero que las contiene y por ser complemento de aquélla, a la vez que un documento de gran interés, como podrá comprobarse.

El texto se encuentra en el manuscrito 489 de la Beinecke Rare Books and Manuscripts Library, biblioteca de la Universidad de Yale. El volu-

qués de Santillana, Madrid, Insula, 1957, págs. 309-312. Una traducción de la De Genealogia Deorum de Boccaccio, probablemente motivada por Santillana, fue definitivamente asignada a un traductor, Martín de Avila, y el Marqués fue identificado como impulsor de la misma; ambos datos fueron recogidos por J. Piccus en su artículo «El traductor español de De Genealogia Deorum», en Homenaje a RodriguezMoñino, Madrid, Castalia, 1966, vol. II, págs. 59-75.

6 Editada por M. Kerkhof en su edición de La Comedieta de Ponza, Groninga, 1977, pág. 510.

7 Para el problema genérico y de definición de proemios, prólogos, cartas, cartasproemios y otros términos vid. A. Poroueras Mayo, El prólogo como género literario. Su estudio en el Siglo de Oro español, Madrid, C. S. I. C., 1957.

8 Hemos realizado una edición del Proemio, trabajo del que damos noticias sobre la labor de ecdótica en el segundo número de Dicenda. 
men es un cancionero copiado hacia la segunda mitad del siglo xvi que perteneció a la colección del bibliófilo inglés Thomas Phillipps y que fue adquirido en subasta por la Universidad de Yale en 1970; puede asegurarse sin ningún género de dudas que ha sido uno de los cancioneros menos conocidos hasta el momento. Está compuesto por obras de Santillana, algunas citas de Macías el Enamorado, unos Proverbios que no son los del Marqués y un texto de Séneca ${ }^{9}$. La carta ocupa los folios 1r-2r a manera de introducción del cancionero, aunque posteriormente se copiaría la carta en la que su sobrino le da las gracias y contesta a la enviada por el Marqués; esta inserción pudo darse en una copia directa del texto enviado por el Marqués o se pudieron añadir en el propio original. Todo parece indicar que el contenido del volumen es el mismo que señala Santillana en la carta:

Quexades vos porque no vos aya embiado los Sonetos y Prouerbios y algunos dichos de sabios o cosas de Séneca que me demandastes;

pero, más tarde, al referirse a sus Sonetos:

yo vos embío solamente media dozena dellos de materias diuersas.

Sin embargo, los Sonetos no aparecen por ninguna parte; este hecho constituye la primera prueba en contra respecto a nuestra afirmación anterior. Otros elementos que pueden contradecir esta idea serían los siguientes: la presencia de otras obras del Marqués a las que no hace referencia en ningún momento dentro de su carta. No sería necesario ni tan siquiera pensar en una copia posterior de las obras, pues éstas habrían sido añadidas después del texto de Séneca, que se encuentra en último lugar; puede suponerse que Santillana simplemente no las nombra. Otra posibilidad sería la de que se hubiesen introducido en la copia del siglo XVI, pero que todos estos poemas no se encontrasen en el ejemplar mandado a Pedro de Mendoza. El siguiente punto oscuro que se podría señalar es el de que los Proverbios del Marqués no aparecen por ninguna parte; pero, como podrá comprobarse, éste no dice que los que manda sean los suyos: con toda seguridad los Proverbios a los que

9 No hemos podido estudiar personalmente el cancionero; para su descripción nos valemos de los datos -algo diferentes unos de otros- ofrecidos por la propia biblioteca de la Universidad de Yale y por B. Dutron en su recién aparecido CatálogoIndice de la Poesía Cancioneril del siglo XV, Madison, 1982, tomo I, págs. 147-48; las siglas que le asigna Dutton en su catalogación son YB2. Las primeras noticias que se dieron de este cancionero -aparte de la que ofrecen algunos catálogos de la colección Phillipps y de la casa Sotheby, que subastó el volumen - fueron las de M. DURAN en su edición de la obra del Marqués, Poesías completas, I, Madrid, Castalia, 1975, pág. 32. 
se refiere son los que aparecen en el cancionero y no los que él mismo compuso para el príncipe Enrique ${ }^{10}$. Por tanto, los únicos problemas so-

10 La información mandada por Yale ofrece el siguiente contenido:
ff. $1-2 r$
ff. $2 v-3 r$
ff. $3 \mathrm{v}-120 \mathrm{r}$
ff. $121 \mathrm{r}-322 \mathrm{r}$
ff. $322 \mathrm{r}-325 \mathrm{r}$
ff. $325 v-327 v$
Letter of Santillana to Señor Pedro de mendoça Señor de almaçan. Reply of Pedro mendoça.
Poems.
Proverbs.
Sayings of the Philosophers.

En cambio, Dutton, tras enumerar las poesías del Marqués que forman el volumen, añade tan sólo:

«26 (121r-327v) (Prosa) Para introducion deste prouerbio: lo tenia en casa ocupado. Proverbios. No son de Santillana», op. cit., pág. 148.

Notas al texto:

1-3. Pedro de Mendoza, Señor de Almazán y Monteagudo, fue, como el propio autor indica y su apellido declara, sobrino del Marqués. Los testimonios acerca de este personaje no son abundantes, pero sí muy confusos. Los datos extraídos de las crónicas más importantes del siglo XV no nos aclaran demasiados puntos: hay algunas noticias en el Memorial de Mosen Diego de Valera o en la Crónica de Alvaro de Luna. Más concreta y aclaradora es la que nos ofrece Diego GuTIÉRREz CoRonel en su Historia genealógica de la casa de Mendoza: «Don Pedro de Mendoza, llamado 'el Fuerte', cuarto señor de Almazán, de Monteagudo y Cascante; guarda mayor del rey D. Enrique IV, en cuyo servicio se halló en la batalla de Olmedo; murió en 1 de septiembre de 1474»; edic. de González Palencia, Cuenca, C. S. I. C., 1946, tomo II, pág. 435. GarrafFa en su Enciclopedia Heráldica y Genealógica hispanoamericana, Madrid, 1952, tomo 56, copia los datos de Gutiérrez Coronel, pero señala como única diferencia que es quinto Señor de Almazán. El hijo de Pedro de Mendoza, Pedro González de Mendoza, es el primer Conde de Monteagudo, como indica Alonso LÓPEz DE HARo en su Nobiliario genealógico de los reyes y títulos de España, Madrid, 1622, tomo II, pág. 46.

Quizás el hecho más importante es que disponemos de poemas de este personaje; así, está la «Respuesta de Pedro de Mendoça a Gomez Manrrique» que aparece en el Cancionero castellano del siglo XV de Foulché-Delbosc, N. B. A. E., 22, Madrid, 1915. Hay ciertos datos biográficos en la poesía inicial de Gómez Manrique, que parece referirse a la prisión sufrida por Pedro de Mendoza. También hay una referencia al mismo hecho en la respuesta del Señor de Almazán. Como ocurre en el resto de los casos, Foulché-Delbosc no da información sobre los Cancioneros en los que se encuentra dicha obra, pero ahora podemos completar rápidamente estas indicaciones a través del Catálogo de Dutron. Así, esta obra aparece en los manuscritos de la Biblioteca de Palacio de Madrid 617 y en el la Nacional de Madrid ms. 7817. Op. cit., págs. 57 y 68 . Hay dos composiciones más de este personaje. Una es la respuesta a un poema de Mena que comienza «Gentil señor dAlmaçan: que las toman do las dan*; el texto de Pedro de Mendoza dice en el primer verso: "Quantos sabios oiran: de probar mas que podran»; se encuentra en el manuscrito 2763 de la Biblioteca Universitaria de Salamanca. $O p$. cit., pág. 139. La última de las obras es el poema «Como hay toque de oro: es oro para dorar», dedicado a su mujer y presente en la 2." edición del Cancionero General. Op. cit., pág. 197.

11. Ms. tempentuosos.

17. Los sonetos no aparecen en el cancionero. Este es otro de los elementos que pueden apoyar la tesis de que el contenido del manuscrito de Yale es diferente en la parte que corresponde al Marqués del que éste envió a su sobrino. Otra posi- 
bre este aspecto son los que proceden de la referencia a los Sonetos; más adelante, en nuestras notas al texto, intentaremos dar alguna solución concreta a este punto. Pero, pasemos directamente al texto, que

bilidad es que fuesen enviados en una copia aparte, aunque esto pudo ocurrir también con el resto de las obras y que inicialmente no formasen un solo volumen. También podría pensarse simplemente en que la copia de Yale no los recoge.

18. «de»; ms. $d u$.

21. Uno de los elementos para la fechación de la carta es la referencia del Marqués al Príncipe, no como cultismo semántico sino en el sentido de «hijo de rey» y «heredero"; se refiere así a Enrique IV, el hijo de Juan II. Este último rey murió el 21 de julio de 1454, con lo que la fecha de composición sería anterior. En todo caso, las cartas se escribieron antes de 1455, año en que muere la mujer del Marqués, a la que alude Pedro de Mendoza en su carta. El término post quem sería el año 1453, época en la que se escribe el Doctrinal de Privados, que forma parte del cancionero. Si suponemos por el contrario que el contenido del cancionero o carpetas era diferente del de este manuscrito de Yale, la fecha inicial estaría marcada por el propio título de Marqués y Conde de fñigo López, ambos otorgados por Juan II el año 1445. Sin embargo, lo más probable es que estas dos cartas se escribiesen el año 1454.

21-25. Sobre la fortuna de Séneca en el siglo XV no hay ninguna duda; véase el trabajo de K. A. BLtHER, Seneca in Spanien. Untersuchungen zur Geschichte der Seneca-Rezeption in Spanien vom 13. bis 17. Jahrhundert, Munich, Franke Verlag, 1969, págs. 42-175. Véase también N. G. RouND, aLas traducciones medievales, catalanas y castellanas de las Tragedias de Séneca», Anuario de Estudios Medievales, 9 (1974 1979), 187-228. En general remitimos a nuestro trabajo, realizado con Ch. B. Faurhaber, B. Dutton, J. NitTi y D. Mackenzie, Bibliography of Old Spanish Texts, 3.a ed. (Madison: Hispanic Seminary of Medieval Studies, 1983).

25-26. La carta, constituida por elementos procedentes de un conocimiento de las artes dictaminis, llega aquí a su punto más importante, la petitio. $\mathrm{K}$. Whinnom llega a señalar en su estudio de la retórica de la Cácel de Amor: *...se escribía una carta únicamente para pedir algo», Dirgo DE SAN PEDRo, Obras completas, II, Madrid, Castalia, 1971, pág. 54.

26-29. Estamos, por supuesto, ante el tema de las armas y las letras, tan unido a la persona del Marqués. Aparte de su propia referencia en el prólogo de los Proverbios y en otros escritos, sus traductores, amigos e historiadores hacen siempre referencia al Marqués y su interés por los libros y la guerra.

37. De las Epistolas a Lucilio se conservan varios ejemplares del siglo Xv. En la biblioteca del Marqués hubo un ejemplar -custodiado en la actualidad en la Biblioteca Nacional de Madrid con la signatura Ms. Res. 7- que contiene las Epistolas y De providentia Dei en italiano (M. ScHIF, La bibliothèque..., págs. 104-11). Otro ejemplar que contiene Obras de Séneca en latín es el actual ms. 10.238 de la B. N. M., que también pudo formar parte de la colección de Santillana (M. ScHIF, id., página 102). Otros manuscritos de la Biblioteca Nacional de Madrid que contienen esta obra son el 10.806 y el 8.368 con la traducción de Fernán Pérez de Guzmán. Para más información, remitimos de nuevo a la Bibliography of Old Spanish Texts, ya citada.

6. Ms. egual nueue.

7. Resolvemos la abreviatura $v$. $m$. como vuestra magnifiçençia por ser el tratamiento que generalmente recibe el Marqués; véase así más arriba, al comienzo, «Muy magnífico señor». Este es también el tratamiento que el Marqués da al Condestable don Pedro de Portugal en el Proemio. Preferimos así este apelativo en lugar de un vuestra merçed.

12-21. En este pasaje reside uno de los puntos más interesantes de la carta de Pedro de Mendoza; se trata del rechazo de los estudios humanísticos y de las letras 
transcribimos sin más cambios que las enmiendas indicadas a pie de página y la inserción de corchetes para nuestras adiciones; la resolución de abreviaturas se señala en cursiva y la acentuación y puntuación siguen los criterios modernos:

Carta del señor Marqués de Santillana al señor Pedro de Mendoça, Señor de Almaçán.

Al muy amado sobrino señor, el Marqués de Santillana e Conde del Real, salud. Yo he muy gran plazer porque vos me çertificades del estado e dispusiçión sa5 ludable de vuestra noble persona e buena casa. E así, por digna compensa, antes de todas cosas vos notifico que, por graçia de Nuestro Señor, yo e todos aquellos que acá vos bien queremos e queredes son en aquel mesmo estado e cuento.

Quexades vos porque no vos aya embiado los Sonetos y Prouerbios y algunos dichos de sabios o cosas de Séneca que me demandastes. No vos marauilledes de

10 qualquier dilaçión o sobreseimiento que los homes fagan de las cosas que mucho querrían, agora agenas, agora propias, como los vientos tempestuosos de muchas partes no çesen. Pero más por sastifazer a vuestra demanda que por la dignidad dellos, e de lo demás que en mí toca, e av́n por ser vna arte peregrina o por ventura no vsada en estas partes, yo vos embío solamente media dozena dellos

15 de materia[s] diuersas, así como embiar se acostumbra entre los amigos y buenos vezinos del fruto de sus vergeles; e con aquella protestaçión: que, si todo ello y los Sonetos bien vos sabían, embiedes por más, ca en todas cosas, qualesquier que sean, me deletaré de vos complazer.

Preguntástesme qué era lo que me paresçía de la trasladaçión de vna epístola

20 de Séneca que me embiastes al tiempo que este verano pasado me partí de Trixueque al señor Prínçipe. De la materia no cale fablar, y baste que sea obra de Séneca; la forma del traduzir me paresçió buena y asaz conforme al seso e letra latina; a dezir vos verdad, quanto a moralidad, dexando las cosas de Sacra Escritura, ciertamente vos non podedes estudiar ninguna mejor cosa nin de ma25 yor vtilidad a la vida presente. E así, muy amado señor sobrino, vos rruego y, por el gran amor que vos he, exorto que, dado del tiempo aquella parte que a las cosas militares y familiares se pertenesçe - como so çierto que lo vos acostumbrades-, non ayades por graue e mal despendido aquello que d'él para lo tal furtáredes. Difusa e muy larga narraçión sería de contar quánto lo tal a toda ma-

30 nera de gentes aproueche, que alli fallaredes en quánto se deuen estimar estos que llamamos bienes de fortuna para que por ellos ayamos de venir a ninguna cosa que de la soberana virtud que es onestad se aparta, e muy muchas otras cosas e documentos çiertamente no poco saludables ni de pequeña autoridad. $\mathbf{E}$ yo, así como este mismo autor dize, bien otorgo, si vos plaze, que de otros libros

35 e ystorias ayades notiçia. Pero esto sea así como espía que va de vn real en otro por saber lo que se faze, más como fuidizo o tránsfuga; e vía este libro de las

en general por cierto sector de la clase alta en esta época. Aunque pueden añadirse más datos al respecto, basta con mirar los ejemplos que ofrece N. G. Round, «Renaissance culture and its opponents in fifteenth century Castilex, Modern Language Review, LVII (1962), págs. 115-38, para tener una buena perspectiva del problema.

20. Ms. Obunbilados. «Estos», ms. esta.

36-39. No queda demasiado clara la referencia de Pedro de Mendoza. 
sus Epistolas a Lucilio sea vuestro compañero e comensal continuo, de lo qual yo fío no vos repentiredes.

El Marqués

Respuesta de Pedro de Mendoça, Señor de Almaçán, al señor Marqués de Santitillana.

Muy magnífico señor, yo, Pedro de Mendoça, Guarda Mayor del Rey nuestro señor e de su consejo, e Señor de las villas de Almaçán e Montagudo.

5 Todos los desta casa somos en aquella abundançia saludable que por los que bien nos quieren deue ser deseada. Lo que sentí en oýr egual nueua de la gran casa de vuestra magnifiçençia no digo, porque de dos cosas será fuerça de yncurrir en vna: si tan biuamente como lo siento lo dixese entera paresçería lisonja, e si lo moderase la verda[d] encubriría. Así que, por evitar tanto viçio, creo 10 el callar seer lo más onesto.

Dize vuestra magnificencia que estoy quexoso por los Sonetos a mí embiados. No so yo el que quexo, mas antes las claras e av́n preclaras obras por vuestra prudençia ordenadas se quexan e tienen tan gran aborresçimiento con toda la mayor parte d'España, como la muger no casta con el temido marido, porque

15 no solamente no se dan a los actos de çiençia mas av́n refuyen dellos e dan lugar que se tome de orín la parte intelectual. E, seyendo del vando de vuestras frutuosas doctrinas, oso dezir que ynsano paresçe no sólo no ser prudente mas avn no desear lo ser; así que con razón deven estar sentidas, porque todos los d'España, allende no descurriendo, no tenemos asistentes de vuestra ilustre persona,

20 éstos para que con ellos nos embiase cosas con que nuestros obnubilados entendimientos despertasen.

Que diuulgue mi poco sentido vuestra magnifiçençia quiere, pues me manda que diga lo que siento de los Sonetos. Por complir vuestro mandado, digo que me acaesçe con ellos lo que con la [Fe]: que no se puede alcançar por razón, alcán-

25 çase por creençia. E para verdaderamente dezir lo que siento dellos, abaste sea cosa de vuestra magnifiçençia, que para en este tiempo es más que lo de Séneca en el suyo. Asimismo dize que esta arte no me paresçerá tanto bien por ser peregrina; e, fablando lo cierto, nosotros somos, señor, los peregrinos e agenos de aquella, ca ella en sí doméstica e graçiosa es. En fin de todo vuestra magnifi-

30 çençia muestra aver plazer en que yo aya por maestro e companero el libro de las Epistolas de Séneca a Luçilio embiadas; en lo yo fazer así mi negoçio fago, pero, por seer vos seruido, fazerlo he con mayor afeçión de aquí adelante. Gusté de la fruta del muy dulçe vergel de vuestra magnifiçençia, el dulçor de la qual me dexó tan fambriento que, neçesitado, embío por más si le pluguiere. Conclu-

35 yendo con el mayor acatamiento e amor que deuemos todos desta pequeña casa, nos encomendamos en merçed vuestra e de mi señora tía. A mis señores primos recompensable recomendaçión embío; bien veo que es yerro, en letra de vuestra magnifiçençia, dar semejante cargo, pero, conosçiendo el deudo que les ha $\mathrm{e}$ amor que deue auer según ellos son, me da esfuerço osadía.

Pedro de Mendoça

ANGBL GOMEz Moreno 